doi: 10.36689/uhk/hed/2018-01-018

\title{
Internet of Things
}

\author{
Petr DOUCEK, Miloš MARYŠKA
}

University of Economics, Prague, Czech Republic

\{Doucek, milos.maryska\} @vse.cz

\begin{abstract}
This paper is devoted to the actual topic the internet of Things. Internet of Things is really frequent topics. The vision of future is a smart world which is interconnecting smart devices enabling qualitatively new services. In this concept, Industry of Things (IoT) plays crucial role. We are dealing with analysis of the most important which are used by companies for providing data from sensors to heir users. At the beginning of the paper we are comparing Internet of Things. The Internet of Things, which contains two parallel streams called Industrial Internet of Things and Industry 4.0 is an evolution of Internet. The Internet of Things in general will be consuming a lot of technical resources nevertheless will be producing a lot of usable information. . Information generated by way of IoT can be used for example in predictive maintenance, improving production cycles, adding addition value to processes based on Just in Time method etc.
\end{abstract}

Keywords: Internet of Things, Industry 4.0, Standards, Technologies.

\section{Introduction}

Internet of Things is really frequent topics. For example amount of links related to Internet of Things is 248 million, Industry 4.0 is 209 million, Industrial Internet of Things 157 millions and when we compare it with amount of links related to Google (11 billions) or Yahoo (2.2 billions) we see, that this new topic has significant number of search.

The vision of future is a smart world which is interconnecting smart devices enabling qualitatively new services. In this concept, Industry of Things (IoT) plays crucial role [16]. Most global companies (56\%) are viewing IoT as a strategic activity [9] where the motivation for implementation is an increase in productivity (24\%), decreasing time to market $(22.5 \%)$ and improving process automation $(21.7 \%)$. The Vodafone IoT Barometer 2016 survey identified that $63 \%$ of businesses will have launched IoT projects in the next year and $76 \%$ of businesses say that IoT will be "critical" to their future success $[15,2]$.

Importance of the IoT is confirmed by IDC which expects, that in 2020 IoT in Central and Eastern Europe will be presented by 1.4 billion of connected things (globally 37 billions) and the market opportunity of IoT will be 24 billion dollars. [9, 15] 
The second view of importance of the Internet of Things for companies is displayed in next figure.

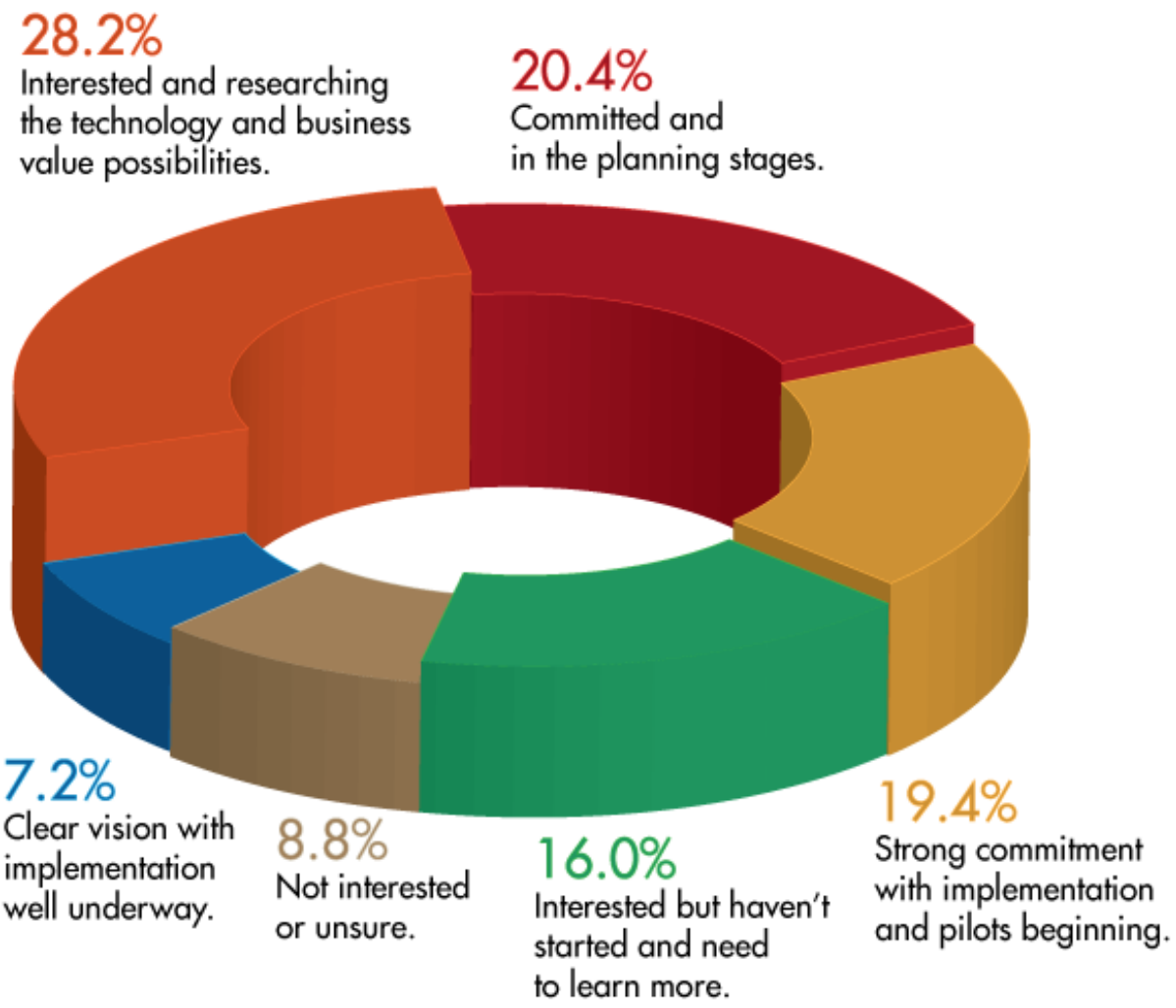

Fig. 1. Importance of Internet of Things and related terms for companies, Source: [2]

Other emerging technologies are overlapping with IoT. Big Data technology could be used to handle massive amounts of data that IoT sensors can produce. Machine learning and advanced analytics are needed to process and analyze IoT data in real time. Voice based human-machine interface is the way how users will interact with devices connected and integrated in IoT world.

\section{Problem Formulation}

Scientist are using words like Internet of Things, Industrial Internet of Things and Industry 4.0 but they are not differentiating among these terms. The aims of this paper is to explain differences between these terms and provides basic information about available technologies, which can be used for harnessing data from sensors etc. 


\section{$3 \quad$ Internet of Things vs Industry 4.0 vs Industrial Internet of Things}

All of those terms are closely related nevertheless there are significant differences especially between Industry 4.0 and Internet of Things and Internet of Things and Industrial Internet of Things which will be explained in next part. Lest start with time differences among these terms which is displayed in next picture.

1999

Internet of Things
2012

Industry Industrial internet of tings

Fig. 2. Evolution of an Internet of Things Source [7]

\subsection{Internet of Things}

IoT can be described as an evolution of Internet in a way that integrates not only mobile devices but also other objects like sensors attached to cars, home appliances, different devices into one interconnected mesh [13]. Smart things integrated in IoT context are able to perform three basic tasks [12]:

- Communication, the ability to wirelessly communicate among themselves, and form ad hoc networks of interconnected objects.

- Identification with a digital name: relationships among things can be specified in the digital domain whenever physical interconnection cannot be established.

- Interaction with the local environment through sensing and actuation capabilities whenever present.

IoT applications were categorized by [4] into four application domains: personal and home, enterprise, utilities and mobile. These domains provide general classification of possible applications of IoT but real business environment usually crosses these domains. Possible applications of IoT in context of industries were identified by [20]. Ability for companies to implement IoT concept and leverage new possibilities can be key to competitive advantage in the future.

Enabling technologies for IoT concept are currently under development or are emerging [10]. Standardization is a little bit lacking in the field of providing much needed standards for interoperability on different layers in IoT architecture [19]. Dynamic environment of evolving enabling technologies creates risks and uncertainty for organization that are willing to adopt IoT technology. Security of IoT environment should also be noted as one system aspect that must be solved robustly in distributed and omnipresent system. [8]

Although we thing that IoT is a new term, this term is old. The first usage of this terms is registered in 1999 and was used by Kevin Ashton during presentation at Procter \& Gamble. [1] 


\subsection{Industry 4.0}

Industry 4.0 is a name for the current trend of automation and data exchange in manufacturing technologies.

Industry 4.0 is a German government initiative, which identifies the fourth industrial revolution which is happening right now. [6] The Industry 4.0 promotes the computerization of manufacturing The 4.0 is based on the name of previous industrial revolution which happened in the mid-twentieth century and linked to information technology.

According to McKinsey [14, 11], Industry 4.0 is "the next phase in the digitization of the manufacturing sector, driven by four disruptions: the astonishing rise in data volumes, computational power and connectivity, especially new low-power wide-area networks; the emergence of analytics and business-intelligence capabilities; new forms of human-machine interaction such as touch interfaces and augmented-reality systems; and improvements in transferring digital instructions to the physical world, such as advanced robotics and 3-D printing."

Industry 4.0 creates what is called a "smart factory". Within the modular structured smart factories, cyber-physical systems monitor physical processes, create a virtual copy of the physical world and make decentralized decisions.

The evolution of industry is illustrated in the next picture.

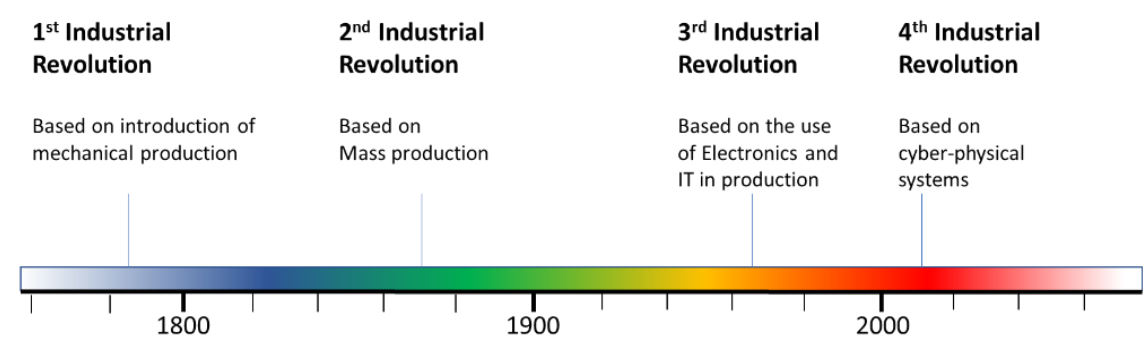

Fig. 3. Evolution of an Industry Source [18, p. 4], [authors]

The Industry 4.0 is based on 4 principles:

- Interoperability

- Information transparency

- Technical assistance

- Decentralized decisions

\subsection{Industrial Internet of Things}

According to the [18], the Industrial Internet of Things (IIoT) promises: "to revolutionize manufacturing by enabling the acquisition and accessibility of far greater amounts of data, at far greater speeds."

In general, Industrial Internet of Things newly contains modern areas like machine learning and big data technology and harnessing of the data from sensors. We are 
including into Industrial Internet of Things services, machines, communication etc. which were used during last years and the new one are enriching it into IIoT.

The target of all of those activities is to achieve so called Smart machines which are better than humans, produce less error than humans, provides real-time and precise information about processing and all activities and producing process.

As [18] says, the IIoT has great potential for quality control, sustainable and green practices, supply chain traceability and overall supply chain efficiency.

The biggest problem with Industrial Internet of Things is a standardization which influence interaction among devices and machines. Standardization is missing and almost each company has its own communication protocol/standard and for companies is really difficult to easily create smart production processes. This situation results in foundation of the nonprofit Industrial Internet Consortium in 2014. Which focuses on standardization that promote open interoperability and the development of common architectures as well as catalyze and co-ordinate priorities and enabling technologies in industry, academia and governments around the Industrial Internet. [3] The Industrial Internet Consortium was formed with support off GE, AT\&T, Cisco, Intel and IBM. The Industrial Internet consortium has over 200 members from private companies and some academic institutions in 12 different countries including India, China and Germany.[20] This distinction of origin and participation, albeit formal, carries with it a profound distinction of vision and approach According to the General Electrics, the benefits of the IioT will be [3]:

- $46 \%$ Percentage of global economy that can benefit from the Industrial Internet

- $100 \%$ Industrial Internet potential impact on energy production

- $44 \%$ Industrial Internet potential impact on global energy consumption

\subsection{Internet of Things vs Industry 4.0 vs Industrial Internet of Things}

Internet of Things. Industrial Internet of Things. Industry 4.0. Based on the text above we already see that these terms are not interchangeable. Each term has a different preconditions, stakeholders, goals and results.

The Internet of things is a network of intelligent devices and objects that harness and share huge amounts of data. The IoT increase automation in homes, schools, stores, and in many industries.

The IIoT is part of concept called as the Internet of Things (IoT). The application of the IoT to the manufacturing industry is called the IIoT (or Industrial Internet or Industry 4.0). The IIoT is part of the Internet of Things. The IIoT is revolutionizing manufacturing by enabling the acquisition and accessibility of far greater amounts of data, at far greater speeds, and far more efficiently than before.

One perspective is to think of the Industrial Internet as connecting machines and devices in industries and helps with predictive maintenance or help to reduce risky situation. The second perspective of the Internet of Things includes consumer-level devices such as heart monitoring fitness bands or smart home appliances.

At the end we can say that Industrial Internet of Things and Industry 4.0 are similar activities which are using Internet of Things as it bases. 
All of these terms are closely connected with Cloud Computing, which means, that an application (from IoT, IIoT, Industry 4.0) is running on computer located remotely.

\section{Conclusions}

The Internet of Things, which contains two parallel streams called Industrial Internet of Things and Industry 4.0 is an evolution of Internet. The Internet of Things in general will be consuming a lot of technical resources nevertheless will be producing a lot of usable information. This information can be used for example in predictive maintenance, improving production cycles, adding addition value to processes based on Just in Time method etc. [15]

Usually users and scientist are using term Internet of Things without any distinction.

Acknowledgements. Paper was processed with contribution of long term support of scientific work on Faculty of Informatics and Statistics, University of Economics, Prague (IP 400040).

\section{References}

1. Ashton, K.: That 'Internet of Things' Thing, http://www.rfidjournal.com/articles/view?4986 last accessed 2017/10/01.

2. A Tale of Two IoT Initiatives: Industry 4.0 vs. the Rest of IoT, http://www.machinedesign.com/motion-control/tale-two-iot-initiatives-industry-40-vsrest-iot, last accessed 2017/10/01.

3. Everything you need to know about the Industrial Internet of Things, https://www.ge.com/digital/blog/everything-you-need-know-about-industrial-internetthings. Last accessed 2017/10/01.

4. Gubbi, J., Buyya, R., Marusic, S., Palaniswami M.: Internet of Things (IoT): A vision, architectural elements, and future directions, Future Gener. Comput. Syst., vol. 29, no. 7, pp. 1645-1660, (2013).

5. Industry 4.0. What is it? https://theleadershipnetwork.com/article/futuremanufacturing/industry-4-0. last accessed: 2017/10/01.

6. Industry 4.0 or Industrial Internet of Things-What's Your Preference? | Automation World. . Available: https://www.automationworld.com/industry-40-or-industrial-internetthings-whats-your-preference last accessed: 2017/10/01].

7. IoT, Industry 4.0, IIoT: http://www.sealevel.com/community/blog/wpcontent/uploads/2016/09/IoTIIoTIndustry_Infographic.jpeg. Last accessed: 2017/10/01.

8. Jing Q., A. Vasilakos V., Wan J., Lu J., and Qiu D., Security of the Internet of Things: perspectives and challenges, Wirel. Netw. vol. 20, no. 8, pp. 2481-2501, (2014).

9. Kalál M., Capturing the IoT opportunity, presented at the IDC IoT Forum 2016, Prague, (2016).

10. Li S., Xu L. D., and Zhao S., The internet of things: a survey, Inf. Syst. Front., vol. 17, no. 2, pp. 243-259, (2015). 
11. Löffler M. and reas Tschiesner, The Internet of Things and the future of manufacturing | McKinsey \& Company, http://www.mckinsey.com/business-functions/digitalmckinsey/our-insights/the-internet-of-things-and-the-future-of-manufacturing. Last accessed: 2017/10/01].

12. Miorandi D., Sicari S., De Pellegrini F., and Chlamtac I., Internet of things: Vision, applications and research challenges, Ad Hoc Netw., vol. 10, no. 7, pp. 1497-1516, (2012)

13. Perera C., Zaslavsky A., Christen P., and Georgakopoulos D., Context Aware Computing for The Internet of Things: A Survey, IEEE Commun. Surv. Tutor. vol. 16, no. 1, pp. 414454, (2014).

14. Ray, B.: IoT vs. Industry 4.0 vs. Industrie 4.0 - What's the Difference? IoT For All, $27-$ (2017).

15. Sládek P. and Maryška M., Internet of things in energy industry, Digitalization in Management, Society and Economy - 25th Interdisciplinary Information Management Talks, 2017, pp. 411-418 (2017).

16. Stankovic J. A., Research Directions for the Internet of Things, IEEE Internet Things J., vol. 1, no. 1, pp. 3-9, (2014).

17. The IoT Barometer, http://www.vodafone.com/business/iot/the-iot-barometer-2016. last accessed: 2017/01/10].

18. What Is IIoT? The Industrial Internet of Things. Available: https://inductiveautomation.com/what-is-iiot. Last accessed: 2017/10/01].

19. Sheng Z., Yang S., Yu Y., Vasilakos A. V., Mccann J. A., and Leung K. K., "A survey on the ietf protocol suite for the internet of things: standards, challenges, and opportunities," IEEE Wirel. Commun., vol. 20, no. 6, pp. 91-98, (2013).

20. Xu L. D., He W., and Li S., "Internet of Things in Industries: A Survey," IEEE Trans. Ind. Inform., vol. 10, no. 4, pp. 2233-2243, (2014). 